\title{
Multiscale analysis of head-on quenching premixed turbulent flames
}

\author{
Umair Ahmed, ${ }^{1, \text { a) }}$ Nguyen Anh Khoa Doan, ${ }^{2}$ Jiawei Lai, ${ }^{1}$ Markus Klein, ${ }^{3}$ Nilanjan \\ Chakraborty, ${ }^{1}$ and Nedunchezhian Swaminathan ${ }^{2}$ \\ 1) School of Engineering, Newcastle University, Newcastle-Upon-Tyne NE1 7RU, \\ United Kingdom \\ ${ }^{2)}$ Department of Engineering, University of Cambridge, Trumpington Street, \\ Cambridge CB2 1PZ, United Kingdom \\ 3) Universität der Bundeswehr München, Werner Heisenberg Weg 39, \\ D-85577 Neubiberg, Germany
}

Multiscale analysis of wall-bounded turbulent premixed flames is performed using three-dimensional direct numerical simulation (DNS) data of flame-wall interaction (FWI). The chosen configuration represents head-on quenching of a turbulent statistically planar stoichiometric methane-air flame by an isothermal inert wall. Different turbulence intensities and chemical mechanism have been analysed. A bandpass filtering technique is utilised to analyse the influence of turbulent eddies of varying size and the statistics of vorticity and strain rate fields associated with them. It is found that the presence of the flame does not alter the mechanism of vortex stretching in turbulent flows when the flame is away from the wall, but in the case of FWI, the mechanism of vortex stretching is altered due to a reduction in the contribution from non-local strain, and the small scales of turbulence start to contribute to flame straining process. The results indicate that small scale eddies do not contribute to the tangential strain rate when the flames are away from the walls, whereas the contribution from the small scales to the tangential strain rate increases when the flame is in the vicinity of the wall. It is also found that the choice of chemical mechanism does not influence the underlying fluid mechanical processes involved in flame-wall interaction.

Keywords: Multiscale analysis, flame-wall interaction, Vorticity, Principal strain rate

a) Electronic mail: umair.ahmed@newcastle.ac.uk 


\section{INTRODUCTION}

Wall-bounded turbulent combustion occurs in many flows of engineering interest (e.g. Spark Ignition (SI) engines, gas turbines) and modelling these flames remains challenging. The turbulence structure is altered by the walls, and the interaction of flame elements with walls leads to modifications of the underlying combustion process ${ }^{1,2,7,16,21,22,37}$. It is well-known that the flame wrinkling and stretching are caused by vortical and straining structures in turbulence ${ }^{36}$, but the interaction of such structures in wall-bounded turbulence is not well understood. Premixed combustion typically occurs at the small scales in the spectrum of turbulence ranging from large energy containing (integral) scales to small viscous (Kolmogorov) scales. However, this whole spectrum of scales does not play a significant role on the flame ${ }^{14,36}$, as the Kolmogorov scales are too weak to stretch the flame ${ }^{38}$. The focus here is on how the small scales of turbulence affect premixed flames in wall-bounded flows.

One of the most common approaches used for reaction rate closure is the flame surface density (FSD) based formulation ${ }^{10}$. Flame stretch, $\kappa$ (a measure of the fractional change in elemental flame surface area, $\delta A$ ) plays an important role in this modelling ${ }^{10,19}$ and it is defined $\mathrm{as}^{8}$ :

$$
\kappa=\frac{1}{\delta A} \frac{d \delta A}{d t}=\left(\delta_{i j}-n_{i} n_{j}\right) S_{i j}+s_{d} \frac{\partial n_{i}}{\partial x_{i}}=a_{T}+2 s_{d} K_{m},
$$

where $\delta_{i j}$ is the Kronecker delta, $n_{i}$ is the $i^{\text {th }}$ component of the flame normal vector $\mathbf{n}=$ $-\nabla c /|\nabla c|$ (where $c=\left(Y_{F}-Y_{F_{R}}\right) /\left(Y_{F_{P}}-Y_{F_{R}}\right)$ is the reaction progress variable where subscripts $R$ and $P$ denote reactants and products respectively), $s_{d}=(D c / D t) /|\nabla c|$ is the displacement speed and $S_{i j}=0.5\left(\partial u_{i} / \partial x_{i}+\partial u_{j} / \partial x_{i}\right)$ is the symmetric strain rate tensor. In Eq. (1), $a_{T}$ represents the tangential strain rate and $K_{m}=0.5 \partial n_{i} / \partial x_{i}$ is the curvature. Flame stretch is also used in other modelling approaches such as the thickened flame model $^{13}$ and the $G$-equation approach ${ }^{36}$. The behaviour of flame stretch is dependent on how the strain rate behaves under the influence of heat release. Hence it is important to understand the dynamics of the underlying strain field and this has been investigated for freely-propagating statistically planar flames in past studies ${ }^{14}$. The presence of walls alters the turbulence and strain rate structure considerably and thus it is worthwhile to investigate the contributions of small scale eddies to the overall stretch and also its implications on the Large Eddy Simulation (LES) modelling of flame-wall interaction (FWI). An attempt is made here to understand the influence of walls on strain rate and vorticity statistics in FWI, 
and their resulting consequences for the near-wall premixed turbulent combustion modelling. This is achieved by interrogating both simple 1-step and multi-step detailed chemistry Direct Numerical Simulation (DNS) data of head-on quenching turbulent premixed flames using multiscale analysis by employing a bandpass filtering technique proposed by Leung et al. ${ }^{35}$.

The paper is organised as follows. The bandpass filtering technique is described in section II. The DNS data used for this analysis is discussed in section III. Results are presented and discussed in section IV and the conclusions are summarised in the final section.

\section{BANDPASS FILTERING}

The bandpass filtering method used here suppresses eddies smaller or larger than $L$, and thus the flame stretch induced by eddies of size $L$ can be extracted. The filtering technique developed in ${ }^{35}$ has been successfully used to investigate freely-propagating statistically planar flames ${ }^{14}$. In the present work, the aforementioned technique is slightly modified to analyse wall-bounded flows. The essential steps in the filtering method along with the modifications are described as follows. The velocity field at a given time is Fourier transformed and then the Fourier coefficients are multiplied by a transfer function $T_{b}(h)=(8 / L)^{1 / 2} h^{2} \exp \left(-h^{2}\right)$ with $h=k L / 2$, where $k=|\mathbf{k}|$ is the magnitude of the

wavenumber. This gives Fourier coefficients of the bandpass filtered velocity field $\hat{\mathbf{u}}_{b}^{L}$. Then the inverse Fourier transform is used to obtain the bandpass filtered velocity in the physical space $\mathbf{u}_{\mathbf{b}}^{\mathbf{L}}$. The no slip condition at the wall is ensured by setting $\mathbf{u}_{\mathbf{b}}^{\mathbf{L}}=0$ at the wall. Elaborate detail of the filtering technique can be found in $\operatorname{Ref}^{35}$. The strain rate and vorticity at scale $L$, denoted by $S_{i j}^{L}$ and $\boldsymbol{\omega}^{L}=\nabla \times \mathbf{u}_{\mathbf{b}}^{\mathbf{L}}$ respectively, are obtained by using the filtered velocity field. Using $S_{i j}^{L}$ in Eq. 1, one obtains $a_{T}^{L}$-tangential strain rate associated with eddies of size $L$. These steps are repeated for several scales to determine the statistical behaviour of $a_{T}^{L}$.

\section{DIRECT NUMERICAL SIMULATION DATA}

The Direct numerical simulation (DNS) database of Chakraborty and co-authors ${ }^{28-32}$ for head-on quenching (HOQ) of statistically planar atmospheric turbulent premixed flames by isothermal inert walls has been considered for this analysis. This database employs a simple 
1-step irreversible as well as a skeletal mechanism (details provided below) for chemistry. A statistically planar flame subjected to forced isotropic unburned gas turbulence employing simple 1-step irreversible chemistry in an inflow/outflow configuration is also analysed to demonstrate the differences between the multiscale analysis of wall bounded and open flames. All the flames investigated in this work are summarised in table I. The simulations in cases A-E have been continued for $t \geq 12 \delta_{z} / s_{L}$ (where $\delta_{z}=\alpha_{T} / s_{L}$ is the Zeldovich flame thickness, $\alpha_{T}$ is the thermal diffusivity of the unburnt mixture and $s_{L}$ is the laminar flame speed), while the simulation in case-F has been continued for $21 \delta_{z} / s_{L}$.

\begin{tabular}{|l|c|c|c|c|c|c|c|c|}
\hline & $u^{\prime} / s_{L}$ & $l / \delta_{t h}$ & $K a$ & $D a$ & Grid Size & Domain Size & Chemistry & Configuration \\
\hline Case-A & 5.0 & 1.67 & 8.65 & 0.33 & $512 \times(256)^{2}$ & $70.6 \delta_{z} \times\left(35.2 \delta_{z}\right)^{2}$ & 1-step & HOQ \\
\hline Case-B & 6.25 & 1.44 & 13.0 & 0.23 & $512 \times(256)^{2}$ & $70.6 \delta_{z} \times\left(35.2 \delta_{z}\right)^{2}$ & 1 -step & HOQ \\
\hline Case-C & 7.5 & 2.5 & 13.0 & 0.33 & $512 \times(256)^{2}$ & $70.6 \delta_{z} \times\left(35.2 \delta_{z}\right)^{2}$ & 1 -step & HOQ \\
\hline Case-D & 9.0 & 4.31 & 13.0 & 0.48 & $512 \times(256)^{2}$ & $70.6 \delta_{z} \times\left(35.2 \delta_{z}\right)^{2}$ & 1 -step & HOQ \\
\hline Case-E & 11.25 & 3.75 & 19.5 & 0.33 & $512 \times(256)^{2}$ & $70.6 \delta_{z} \times\left(35.2 \delta_{z}\right)^{2}$ & 1 -step & HOQ \\
\hline Case-F & 7.5 & 2.5 & 13.0 & 0.34 & $(256)^{3}$ & $\left(35.2 \delta_{z}\right)^{3}$ & 1 -step & HOQ \\
\hline Case-G & 7.5 & 2.5 & 13.0 & 0.34 & $(256)^{3}$ & $(7.65 \mathrm{~mm})^{3}$ & Skeletal & HOQ \\
\hline Case-H & 7.5 & 2.5 & 13.0 & 0.34 & $800 \times(400)^{2}$ & $140.5 \delta_{z} \times\left(70.18 \delta_{z}\right)^{2}$ & 1-step & Inflow/Outflow \\
\hline
\end{tabular}

TABLE I. Initial turbulence parameters away from the wall for head-on quenching cases A-G, and forcing parameters for the planar flame simulation in case- $\mathrm{H}$.

The simple 1-step irreversible chemistry simulations have been performed using a threedimensional compressible DNS code, SENGA ${ }^{23}$, which employs high-order finite-difference (10th order for internal points and gradually decreasing to 2nd order at the non-periodic boundaries) and Runge-Kutta (3rd order explicit) schemes for spatial differentiation and time advancement, respectively. It solves the governing equations of mass, momentum, energy and reaction progress variable $c$ in non-dimensional form ${ }^{23}$. The thermo-physical properties such as dynamic viscosity, thermal conductivity, and density-weighted mass diffusivity are taken to be constant and independent of temperature. Standard values of Zeldovich number $\beta_{z}=T_{a}\left(T_{a d}-T_{R}\right) / T_{a d}^{2}$ (where $T_{a}$ is the activation temperature and $T_{a d}$ is the adiabatic flame temperature), Prandtl number $\operatorname{Pr}$ and ratio of specific heats $\gamma$ (i.e. $\beta_{z}=6.0, \operatorname{Pr}=0.7$ and $\left.\gamma=1.4\right)$ are used where the Lewis numbers of all the species 
are taken to be unity. All the simulations have been run until the mean, maximum and minimum values of wall heat flux converged following the flame quenching. Note that the domain size for case-F is smaller in the $x$ direction and the flame interacts with the wall at an earlier time when compared with cases A-E.

The DNS of HOQ using a skeletal chemical mechanism has been conducted using the three-dimensional compressible code SENGA2 ${ }^{9}$. This code also employs a 10 th order central difference scheme for the internal grid points, but unlike SENGA the order of differentiation gradually decreases to a one-sided 4th order scheme at the non-periodic boundaries. The time advancement is carried out using an explicit low-storage 4th order Runge-Kutta scheme. A skeletal chemical mechanism involving 16 species and 25 reactions (among these 10 reactions are reversible) for atmospheric pressure combustion of methane air mixture ${ }^{42}$ is used to solve for chemistry in the detailed chemistry simulation. The thermo-physical properties such as viscosity and thermal conductivity are taken to be functions of temperature, and CHEMKIN polynomials have been used to account for temperature dependence for these physical properties. A mixture-averaged transport approach is adopted, and Soret and Dufour effects are considered in heat and mass transfer.

In the head-on quenching configuration a rectangular box is considered as listed in table I for different cases. The simulation domain is discretised using a uniform Cartesian grid (see table I for grid sizes for different cases). The mesh used in the simulations ensures 10 grid points across the thermal flame thickness for single step chemistry cases and 15 grid points across the thermal flame thickness for the detailed chemistry case, $\delta_{t h}=\left(T_{a d}-T_{R}\right) / \max |\nabla T|_{\mathrm{L}}$, where the subscript ' $\mathrm{L}$ ' refers to unstrained laminar flame quantities. Figures 1-2 exemplarily show the flow configuration for the simple and detailed chemistry simulations. The instantaneous iso-surfaces of the progress variable are shown at different times in figures 1-2 for case-E and case-G respectively. Note that the progress variable in case- $\mathrm{G}$ is calculated by using the normalised methane mass fraction. An isothermal inert no-slip wall at reactant temperature, $T_{R}$, is specified at $x=0$ and the wall normal mass flux is specified to be zero. The boundary opposite to the wall is taken to be partially non-reflecting and is defined using the Navier-Stokes characteristic boundary condition technique $^{44}$, whereas the transverse boundaries are taken to be periodic. The velocity field is initialised using a homogeneous isotropic field of turbulent velocity fluctuations, which is generated using a pseudo-spectral method ${ }^{40}$ following the Batchelor-Townsend spectrum ${ }^{4}$, 
but the velocity components at the wall $u_{1}, u_{2}$ and $u_{3}$ are specified to be zero to ensure a no-slip condition. This field is allowed to evolve for an initial eddy turn-over time before interacting with the flame. This time is sufficient for evolving the flow field and ensuring that there is no influence of the initial condition on vorticity and enstrophy ${ }^{30}$. Following that, a steady unstrained planar laminar premixed flame solution is used to initialise the reactive field. The flame is initially placed away from the wall such that the non-dimensional temperature $T=\left(\widehat{T}-T_{R}\right) /\left(T_{a d}-T_{R}\right)=0.9$ (where $\widehat{T}$ is the instantaneous dimensional temperature) isosurface remains sufficiently away from the wall. The distance ensures that the flame gets enough time to evolve before interacting with the wall. The initial values of normalised root-mean-squared (rms) turbulent velocity fluctuation $u^{\prime} / s_{L}$, longitudinal integral length scale to thermal flame thickness ratio $l / \delta_{t h}$, Damköhler number $D a=l s_{L} / u^{\prime} \delta_{t h}$, and Karlovitz number $K a=\left(u^{\prime} / s_{L}\right)^{1.5}\left(l / \delta_{t h}\right)^{-0.5}$ away from the wall are summarised in table I; the heat release parameter $\tau=\left(T_{a d}-T_{R}\right) / T_{R}$ is taken to be 6.0 for all the head on quenching simulations. The simulations for turbulent cases have been carried out until a time when the maximum and minimum values of wall heat flux become identical following the flame quenching. The simulation time remains different for different cases, but the simulations for all cases were continued for a time, which corresponds to 21, 30, 21, 15, 21, 15 and 9 initial eddy turn-over times (i.e. $l / u^{\prime}$ ) for cases A-G respectively.

In the planar flame calculation (case-H) an inflow/outflow configuration is used and the turbulence upstream of the flame is forced using a modified form of Lundgren's forcing developed by Klein et al. ${ }^{26}$ to maintain the specified values of turbulence intensities and integral length scales upstream of the flame. A rectangular domain as shown in figure 3 is considered and is discretised using a uniform Cartesian grid (see table I for grid and domain size). The current grid resolution ensures 10 grid points across the thermal flame thickness $\delta_{t h}$ and it also ensures that the Kolmogorov scale is resolved in at least 2 grid points at the turbulence intensity considered in this paper. In this case a laminar flame with the heat release parameter $\tau=6$ is initialised in the domain and the turbulence is forced up to the desired level as reported in table I. In this case the boundary condition in the $x$ direction is treated as inflow at $x=0$ and outflow at $x=70.18 \delta_{z}$ and is specified using the NavierStokes characteristic boundary condition technique ${ }^{44}$, while the boundaries in the transverse direction are treated as periodic. The simulation is run for 6 eddy turn over times before extracting the statistics for analysis. 


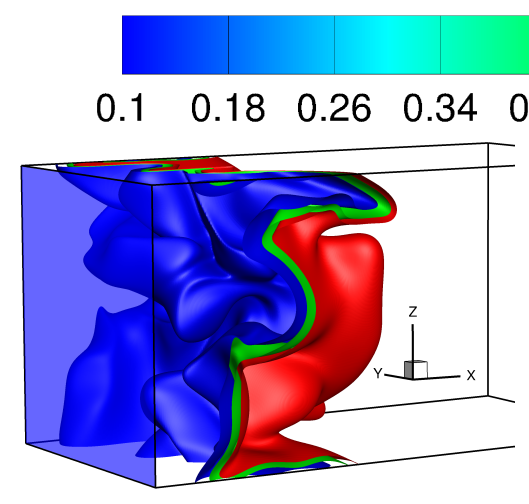

(a) $\delta_{z} / s_{L}$

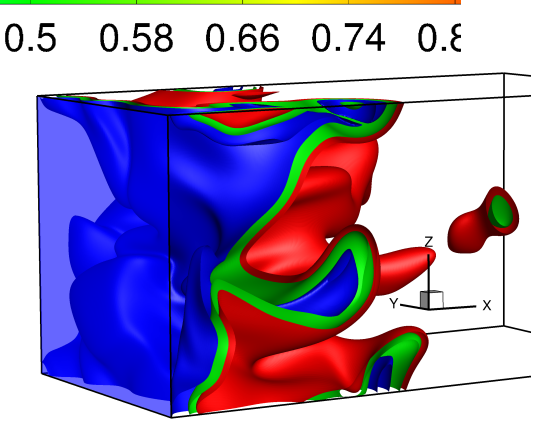

(b) $2 \delta_{z} / s_{L}$

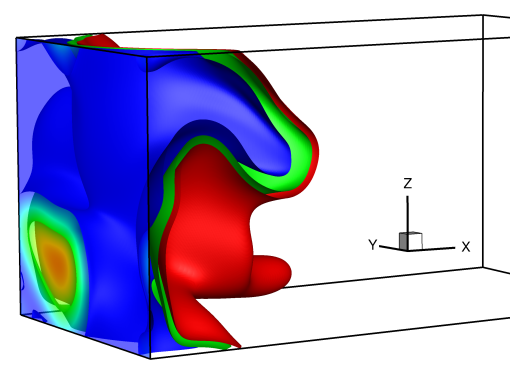

(c) $4 \delta_{z} / s_{L}$

FIG. 1. Flow configuration for the head-on quenching flames case A-E. Three different time instants for case-E are shown. The iso-surfaces represent progress variable. The semi-transparent surface at $x=0$ represents the wall.

In the post-processing of the DNS data, the time (Reynolds) averaged quantities (denoted by $\bar{\lambda}$ ), Favre averaged quantities (denoted by $\widetilde{\lambda}=\overline{\rho \lambda} / \bar{\rho}$ ) and Favre fluctuations (denoted by $\left.\lambda^{\prime \prime}=\lambda-\tilde{\lambda}\right)$ have been ensemble averaged on the planes in the transverse $(y$ and $z$ ) directions at a given $x$ location. The statistical convergence has been ensured by establishing that halving the number of instantaneous samples used for obtaining the statistics did not have any noticeable difference (the maximum difference in the mean values remains less than $1 \%$ ).

\section{RESULTS AND DISCUSSION}

Figures 4-6 show the distributions of Favre-mean non-dimensional temperature $\widetilde{T}$, progress variable $\widetilde{c}$ and turbulent kinetic energy $\widetilde{k}=\widetilde{u_{i}^{\prime} u_{i}^{\prime}} / 2$ in the wall normal direction at different times in case-A, case-C, case-E and case-G respectively. Note that the time instants for case-G are slightly different from other cases due to the differences in the thermo-chemistry used in this case. The overall behaviour of $\widetilde{T}$ in case-G is consistent with 


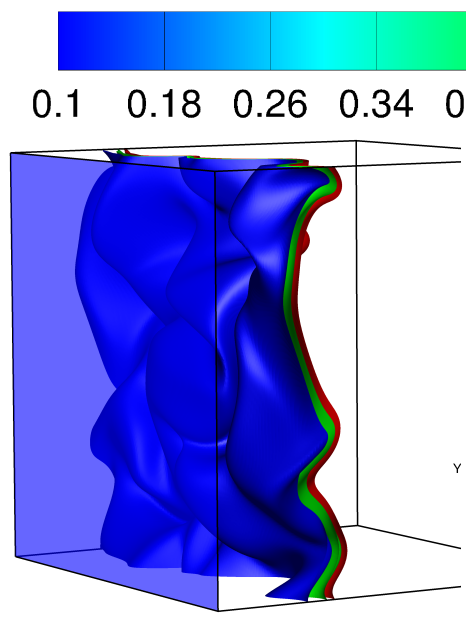

(a) $\delta_{z} / s_{L}$

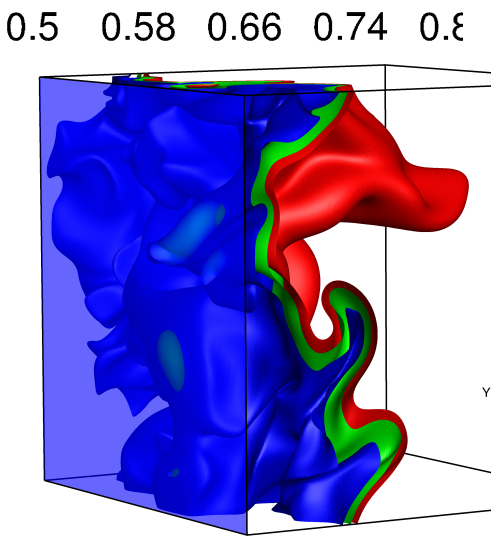

(b) $2.4 \delta_{z} / s_{L}$

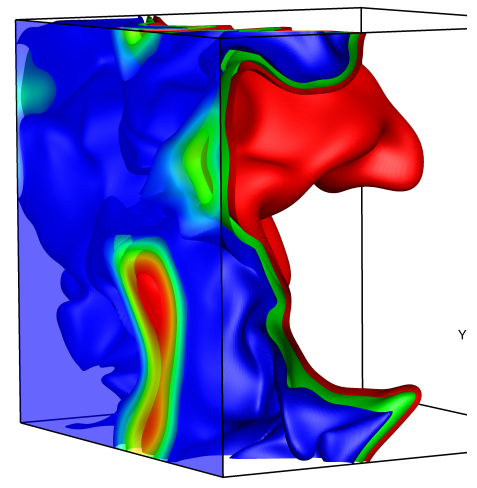

(c) $8.2 \delta_{z} / s_{L}$

FIG. 2. Flow configuration for the head-on quenching flames case-F and case-G. Three different time instants for case- $\mathrm{G}$ are shown. The iso-surfaces represent progress variable. The semitransparent surface at $x=0$ represents the wall.

the behaviours observed for cases A, C and E. It is important to recognise that the domain size of case- $\mathrm{G}$ is different from cases $\mathrm{A}, \mathrm{C}$ and $\mathrm{E}$ as mentioned in table 1. This difference in domain size leads to differences in the instantaneous flame wrinkling behaviour because the turbulent flow realisations are different between case- $\mathrm{G}$ and case- $\mathrm{C}$ while the statistical properties of the turbulent flow are similar. When case-G and case-F are plotted side by side as shown in figure-2 of Ref..$^{32}$ there is no marked difference between the single step and detailed chemistry cases. It can be noticed that the flame behaviour varies with the level of turbulence encountered by the flame. In the case of low turbulence intensity the flame interacts with the wall at a much later time when compared with the higher turbulence intensity $\operatorname{cases}^{28,29,31}$. This happens due to greater extent of flame wrinkling in the higher 


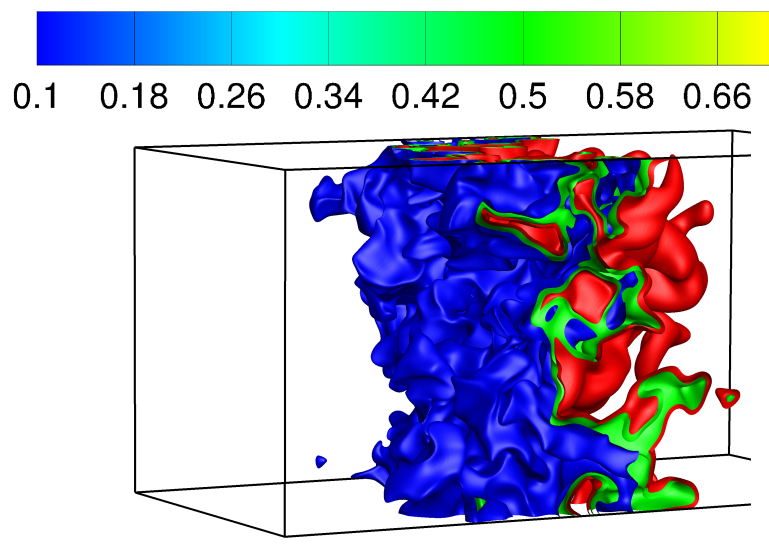

FIG. 3. Flow configuration for the forced turbulence planar flame (case-H) . The iso-surfaces represent progress variable.

turbulence intensity cases. Thus, the flame quenching initiates earlier for cases with higher $u^{\prime} / s_{L}$. This can be further explained in terms of the normalised flame surface area $\left(A_{T} / A_{L}\right)$ and normalised values of turbulent flame speed $\left(S_{T} / s_{L}\right)$ and a detailed discussion for headon quenching flames can be found $\mathrm{in}^{29,33}$ (see table $2 \mathrm{in}^{29,33}$ ). It should be noted here that the turbulence kinetic energy remains at a reasonable level at the times when the data is extracted from the simulations as shown in figure 6 . This implies that the data for head-on quenching flames is suitable for multiscale analysis at the time instances considered in this work.

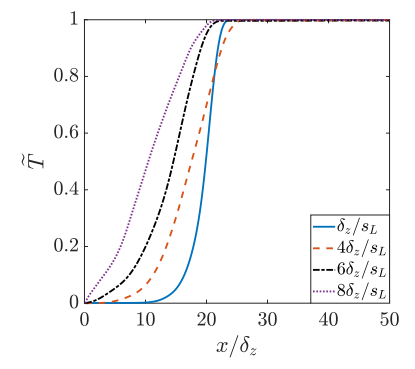

(a)case-A

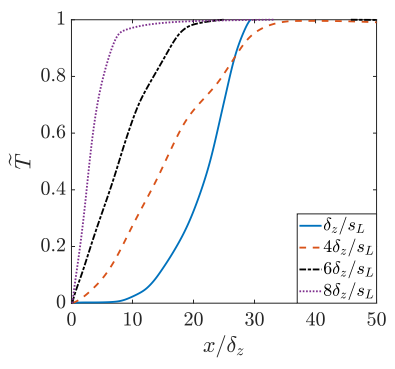

(b) case-C

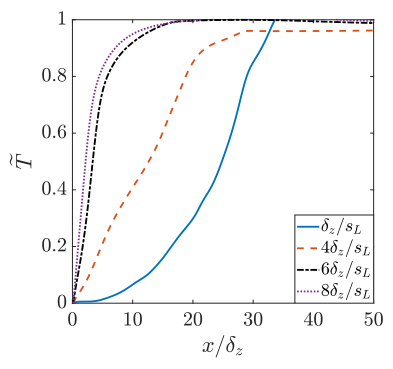

(c)case-E

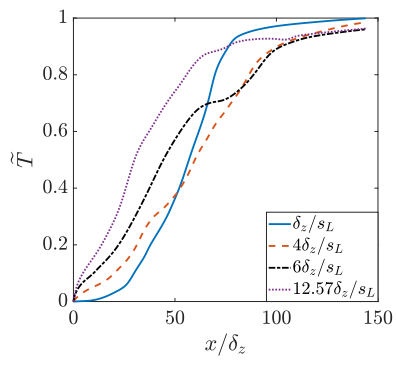

(d)case-G

FIG. 4. Distribution of Favre-mean temperature $(\widetilde{T})$ in the wall normal direction for the head-on quenching flames. 


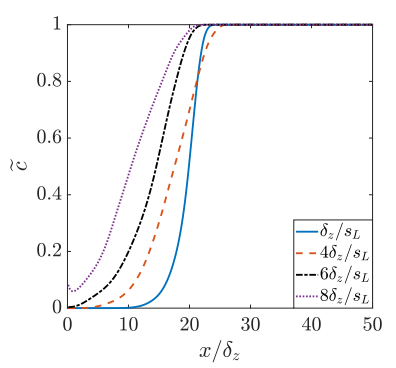

(a)case-A

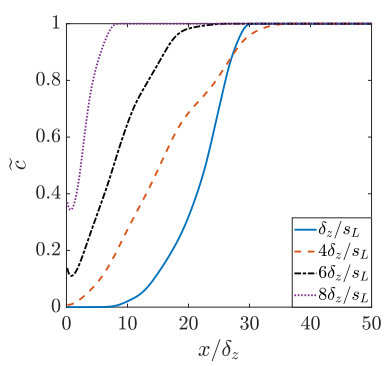

(b) case-C

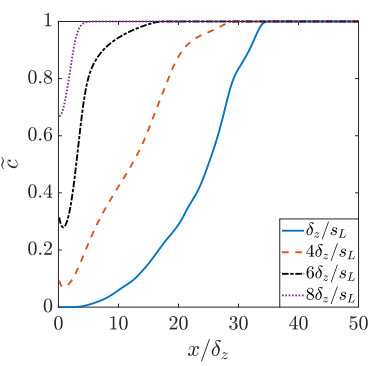

(c)case-E

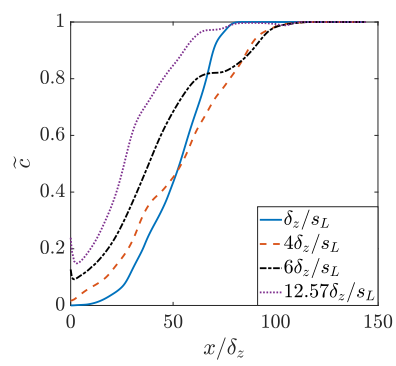

(d)case-G

FIG. 5. Distribution of Favre-mean progress variable $(\widetilde{c})$ in the wall normal direction for the head-on quenching flames.

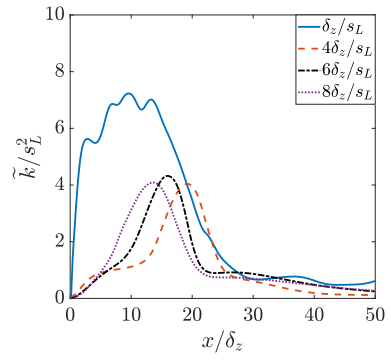

(a)case-A

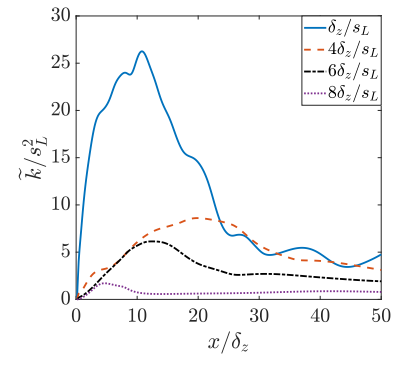

(b) case-C

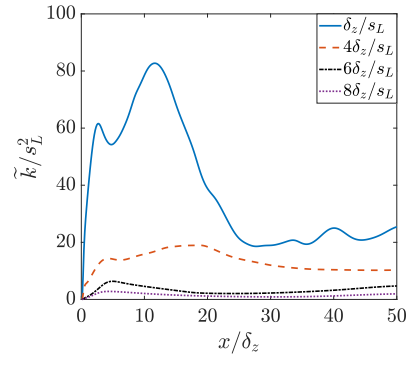

(c)case-E

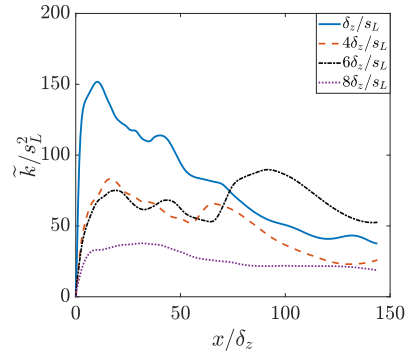

(d)case-G

FIG. 6. Distribution of Favre-mean turbulent kinetic energy $(\widetilde{k})$ in the wall normal direction for the head-on quenching flames.
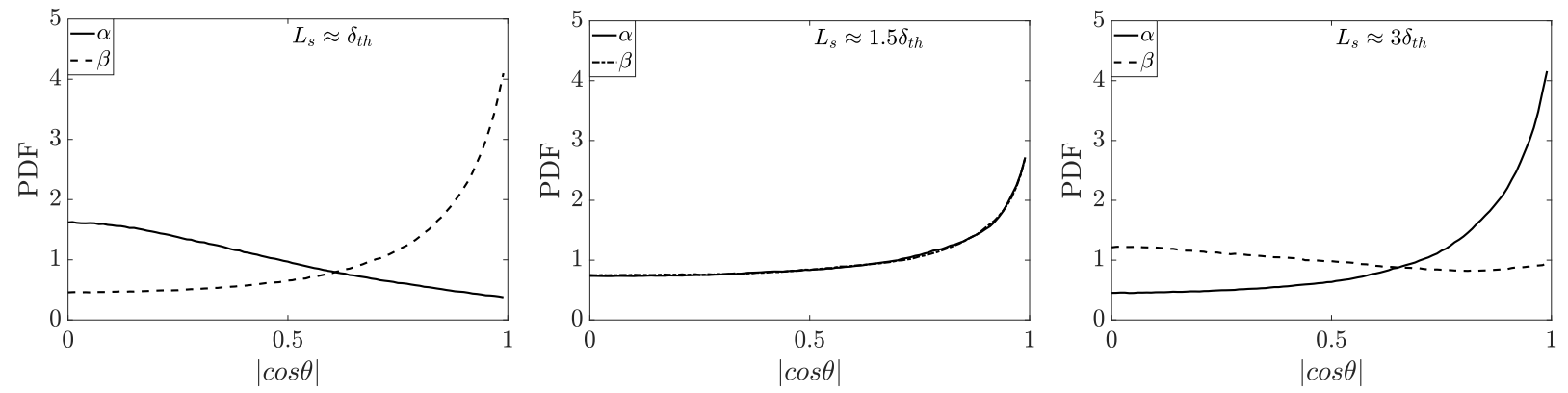

FIG. 7. PDF of the magnitudes of direction cosines between vorticity at $L_{\omega}=\delta_{t h}$ and principal strain rate at $L_{s}$ for the planar flame case (case-H).

\section{A. Influence of combustion on vortex stretching}

It is well-known that vortex-stretching plays an integral role in the transfer of energy across eddies of different scales in turbulent flows. According to the classical picture of turbulence, eddies of smaller size are generated by the breakup of larger eddies due to 

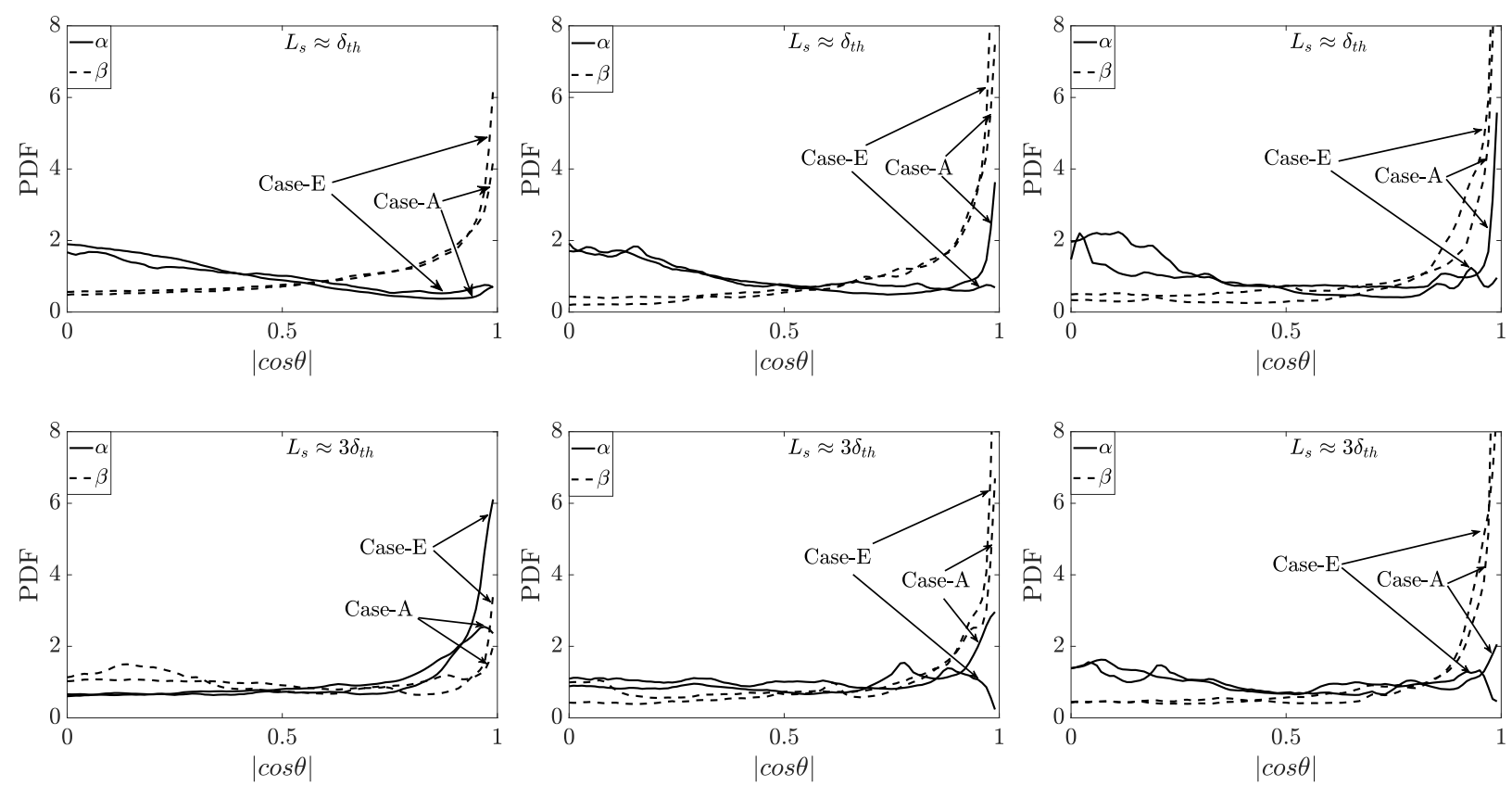

FIG. 8. PDF of the magnitudes of direction cosines between vorticity at $L_{\omega}=\delta_{t h}$ and principal strain rate at $L_{s}$ for simple chemistry head-on quenching flames (case A-E). Three different times $\delta_{z} / s_{L}$ (left), $4 \delta_{z} / s_{L}$ (middle) and $6 \delta_{z} / s_{L}$ are shown.

stretching. This mechanism has been investigated by examining the alignment between the vorticity vector $(\boldsymbol{\omega})$ and the principal directions of the strain rate tensor. The enstrophy production by vortex-stretching can be expressed as $\omega_{i} S_{i j} \omega_{j}=\omega_{i}^{2}\left(\alpha \cos ^{2} \theta_{\alpha}+\beta \cos ^{2} \theta_{\beta}+\right.$ $\gamma \cos ^{2} \theta_{\gamma}$ ), where $\alpha, \beta$ and $\gamma$ are the extensive, intermediate and compressive eigenvalues and $\theta_{i}$ represents the angle between $\boldsymbol{\omega}$ and the eigenvector associated with the principal strain rate $i^{3}$. Previous analyses demonstrated that the vorticity aligns either with $\alpha$ or the positive part of $\beta$ to produce enstrophy through stretching as shown in $^{3}$ for non-reacting flows and in ${ }^{5,6,11,17,30,43}$ for turbulent premixed flames. Jimenez ${ }^{24}$ argued that the principal strain rates with the largest two magnitudes remain in the equatorial plane of the vortex, which has considerable large vorticity in comparison to the background flow. As a result, the instantaneous vorticity aligns with the intermediate strain rate direction. The physical explanation and the modelling implications of the vorticity vector aligning with different eigendirections of the strain rate tensor in premixed turbulent combustion are discussed in detail by Chakraborty ${ }^{11}$, where it is demonstrated that the mean contribution of the vortexstretching term remains positive irrespective of the nature of vorticity alignment with local 

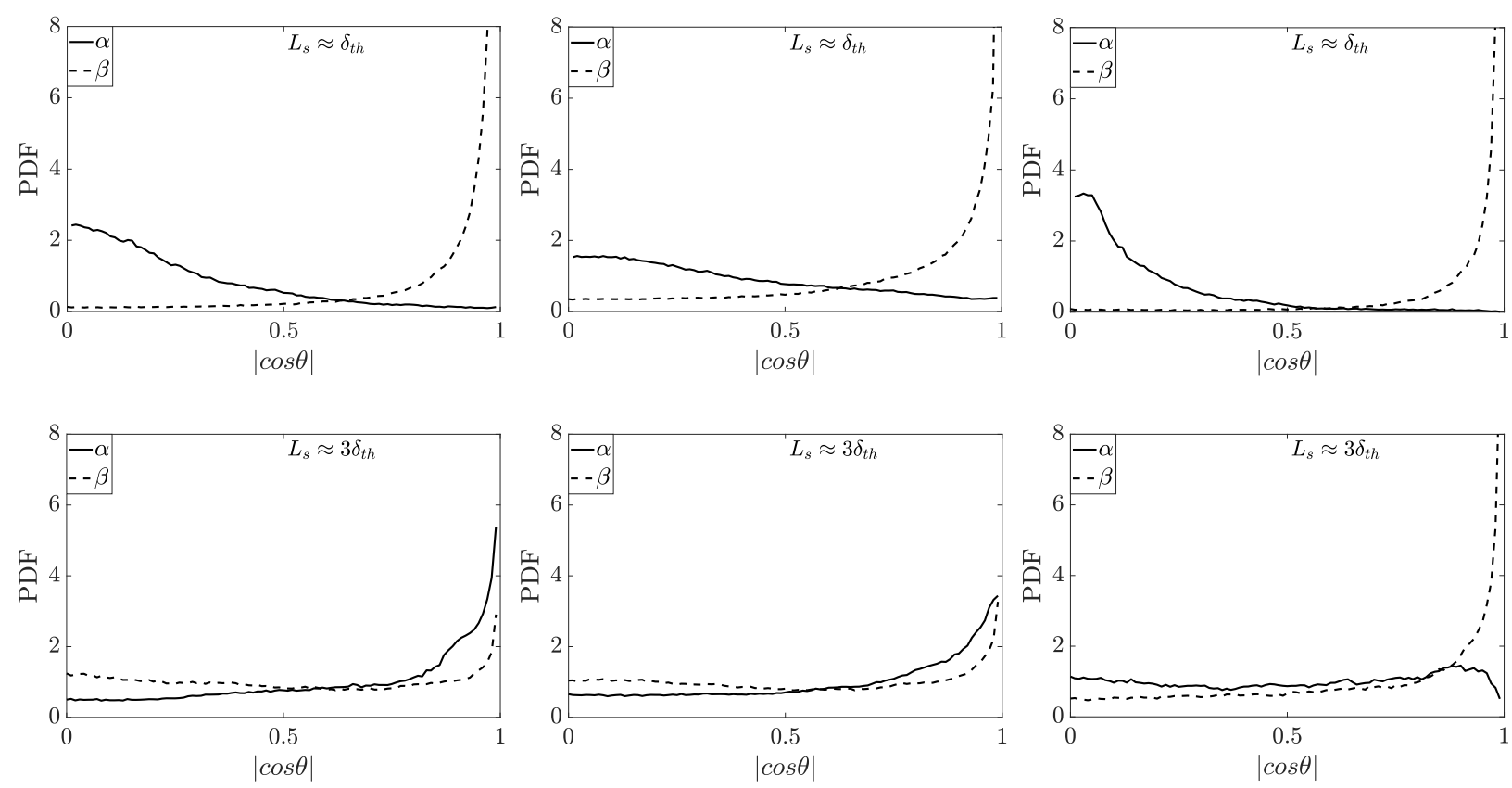

FIG. 9. PDF of the magnitudes of direction cosines between vorticity at $L_{\omega}=\delta_{t h}$ and principal strain rate at $L_{s}$ for the detailed chemistry head-on quenching flame (case-G). Three different times $\delta_{z} / s_{L}$ (left), $2.4 \delta_{z} / s_{L}$ (middle) and $8.2 \delta_{z} / s_{L}$ (right) are shown.

principal strain rate directions.

Leung et al. ${ }^{35}$ have used the bandpass filtering method to demonstrate that the vorticity associated with length $L_{\omega}$ aligns with $\alpha$ for scales $L_{s}>L_{\omega}$ (where $L_{s}$ is the scale used to calculate the filtered strain rate and $L_{\omega}$ is the scale used to obtain vorticity) and the enstrophy production is highest for $L_{s} \approx 4 L_{\omega}$. Similar behaviour has been observed by Doan et al. ${ }^{14}$ for premixed flames subjected to decaying isotropic turbulence.

Figure 7 shows the probability density functions (PDFs) of $\left|\cos \theta_{i}\right|$ for the most extensive, $\alpha$, and intermediate, $\beta$, principal strain rate for the planar flame case (case-H) using the parameters $L_{\omega}=\delta_{t h}$ and $\delta_{t h} \leq L_{s} \leq 3 \delta_{t h}$. Note that the scales smaller than $\delta_{t h}$ are unable to stretch the flame ${ }^{38}$, and in order to understand the influence of stretch induced by scales larger than $\delta_{t h}, L_{\omega}$ is chosen such that it is equal to the thermal flame thickness. Furthermore, the consequences of changing $L_{\omega}$ are shown in figure 3 of Ref. ${ }^{14}$ and it is demonstrated that if $L_{\omega}$ less than or greater than $\delta_{t h}$ is used all the vortical information related to the flame is lost. In this work the PDFs are computed across the entire flame as done by Doan et al. ${ }^{14}$. Note that a preferential alignment of $\boldsymbol{\omega}$ with $\beta$ is obtained for small filter size but the 
alignment switches to $\alpha$ as the filter size is increased this implies that $\boldsymbol{\omega}$ aligns with $\alpha$ for eddies larger than the vortical structure and the alignment with $\beta$ occurs when $L_{s} \leq L_{\omega}$. These results are consistent with the earlier findings of Doan et $\mathrm{al}^{14}$. The current analysis focuses on the behaviour of the aforementioned alignment for wall-bounded premixed flames. The PDFs of $\left|\cos \theta_{i}\right|$ for the most extensive, $\alpha$, and intermediate, $\beta$, principal strain rates are shown in figure 8 for $L_{\omega}=\delta_{t h}$ and $\delta_{t h} \leq L_{s} \leq 3 \delta_{t h}$ for case-A and case-E. Note that a preferential alignment of $\boldsymbol{\omega}$ with $\beta$ is obtained for small filter size but the alignment switches to $\alpha$ as the filter size is increased at early times (e.g. $t=\delta_{z} / s_{L}$ ). The alignment of $\boldsymbol{\omega}$ with $\beta$ implies that the local effects of strain are influencing the flame structure (i.e. $L_{s} \leq L_{\omega}$ ), whereas, when the vorticity vector aligns with $\alpha$ for larger filter sizes, it implies that the non-local contributions in the strain field are also influencing the flame structure. Figure 8 shows that the vorticity aligns with $\beta$ for $t=4 \delta_{z} / s_{L}$ regardless of the variation in filter size in the case of high turbulence intensity (case-E). This is due to the fact that the flame is interacting with the wall, which can be substantiated by examining the distributions of $\tilde{c}$ in figure 5 at these times. Also note that a drop in the values of $A_{T} / A_{L}$ and $S_{T} / s_{L}$ (not shown here but seen in table $2 \mathrm{in}^{29,33}$ ) is seen once the flame starts to interact with the wall and further discussion in this regard can be found in ${ }^{29,33}$. The vorticity generation at the wall weakens with the progress of flame-wall interaction, and consequently this removes one of the sources of non-local (background) strain which is one of the major contributors to the alignment between $\boldsymbol{\omega}$ and $\alpha^{18,35}$. Figure 9 shows the PDF for the magnitude of the direction cosine between $\boldsymbol{\omega}$ and two of the principal strain rate directions at three different times for the detailed chemistry case (case-G). It can be seen from figure 9 that the vorticity alignment statistics are similar to those of case-A and case-E. In the near-wall region $\boldsymbol{\omega}$ aligns with $\beta$ regardless of the filter width, while at locations away from the wall $\boldsymbol{\omega}$ aligns with $\alpha$ for eddies larger than the vortical structure. Note that the alignment behaviour for the principal directions of the strain rate and vorticity remains similar for all the head-on quenching cases regardless of the choice of chemistry used in the DNS calculations. These findings are consistent with the earlier results reported $\mathrm{in}^{32}$.

The probability, $P$ for $0.92 \leq\left|\cos \theta_{\alpha}\right| \leq 1$ is investigated as a function of $L_{s} / L_{\omega}$ for all the flames considered in this study (note that different values for the lower bound of $\left|\cos \theta_{\alpha}\right|$ were examined and no difference was observed in the overall trends of $P$ ). Figure 10 shows that in the planar flame case, the perfect alignment occurs at $2 \leq L_{s} / L_{\omega} \leq 4$, which is consistent 


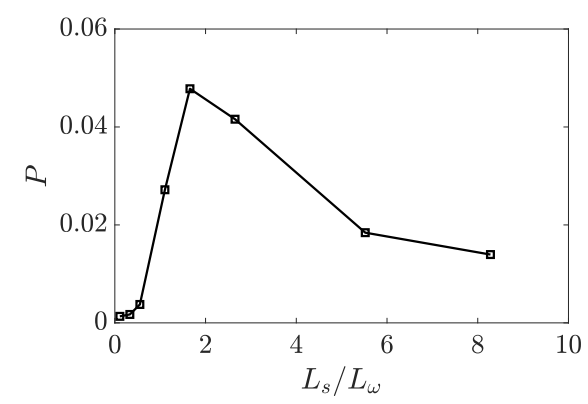

FIG. 10. Probability of perfect alignment between the vorticity at $L_{\omega}$ and extensive principal direction $\alpha$ of the strain rate for the planar flame (case-H).
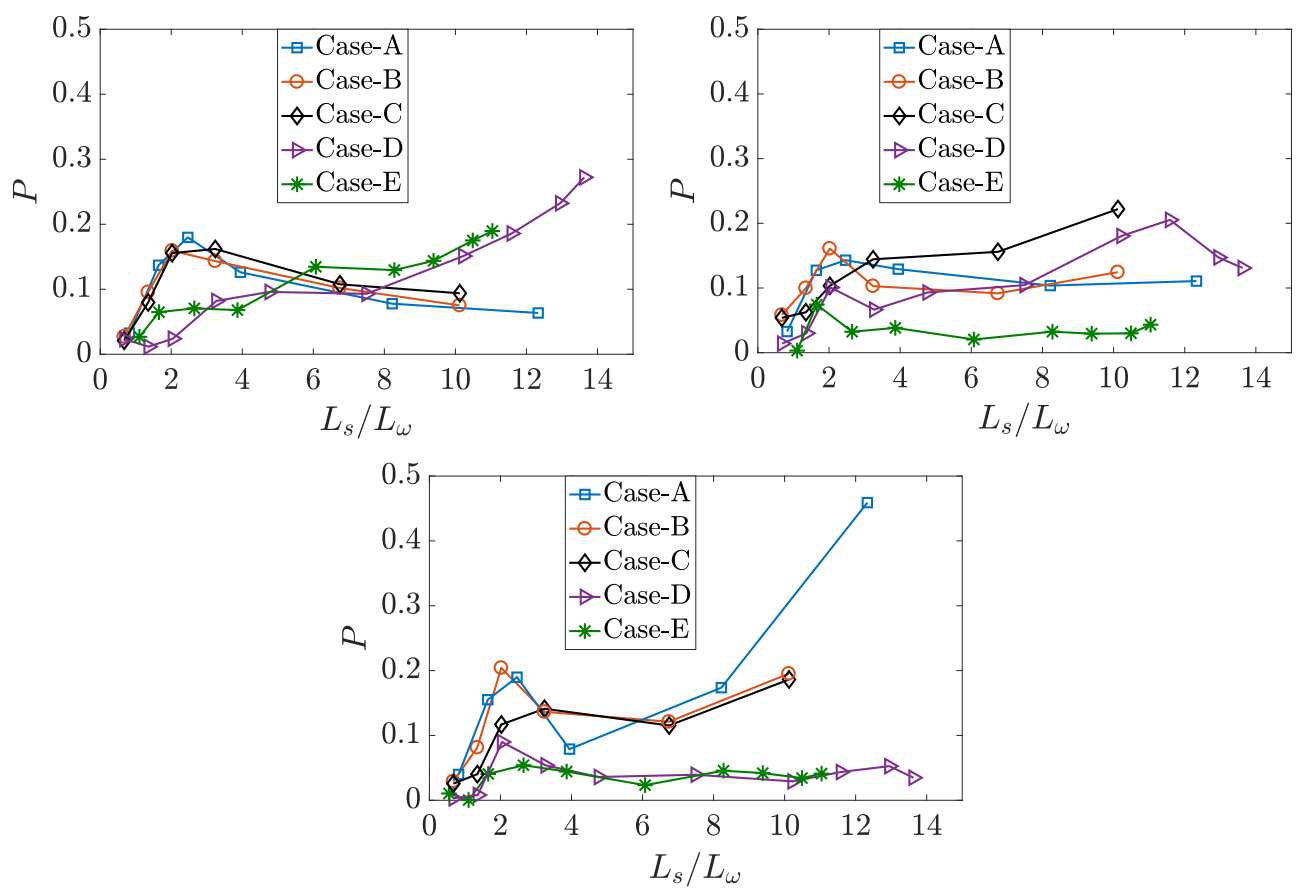

FIG. 11. Probability of perfect alignment between the vorticity at $L_{\omega}$ and extensive principal direction $\alpha$ of the strain rate. Three time instances are shown for the head-on quenching flame with simple chemistry; $\delta_{z} / s_{L}$ (top left), $4 \delta_{z} / s_{L}$ (top right) and $6 \delta_{z} / s_{L}$ (bottom).

with the earlier findings of Doan et $\mathrm{al}^{14}$. In the head-on quenching cases A-C at $t=\delta_{z} / s_{L}$, the maximum probability for alignment occurs approximately at a similar ratio of $L_{s} / L_{\omega}$ found in the planar flame case. In the case of higher turbulence intensity flames (case-D and case-E), the probability of alignment is much larger as these flames have already started to interact with the wall at $t=\delta_{z} / s_{L}$ (see figure 5 ) and consequently the vorticity field is altered in this region. Similar behaviour is observed in the lower turbulence intensity flames 

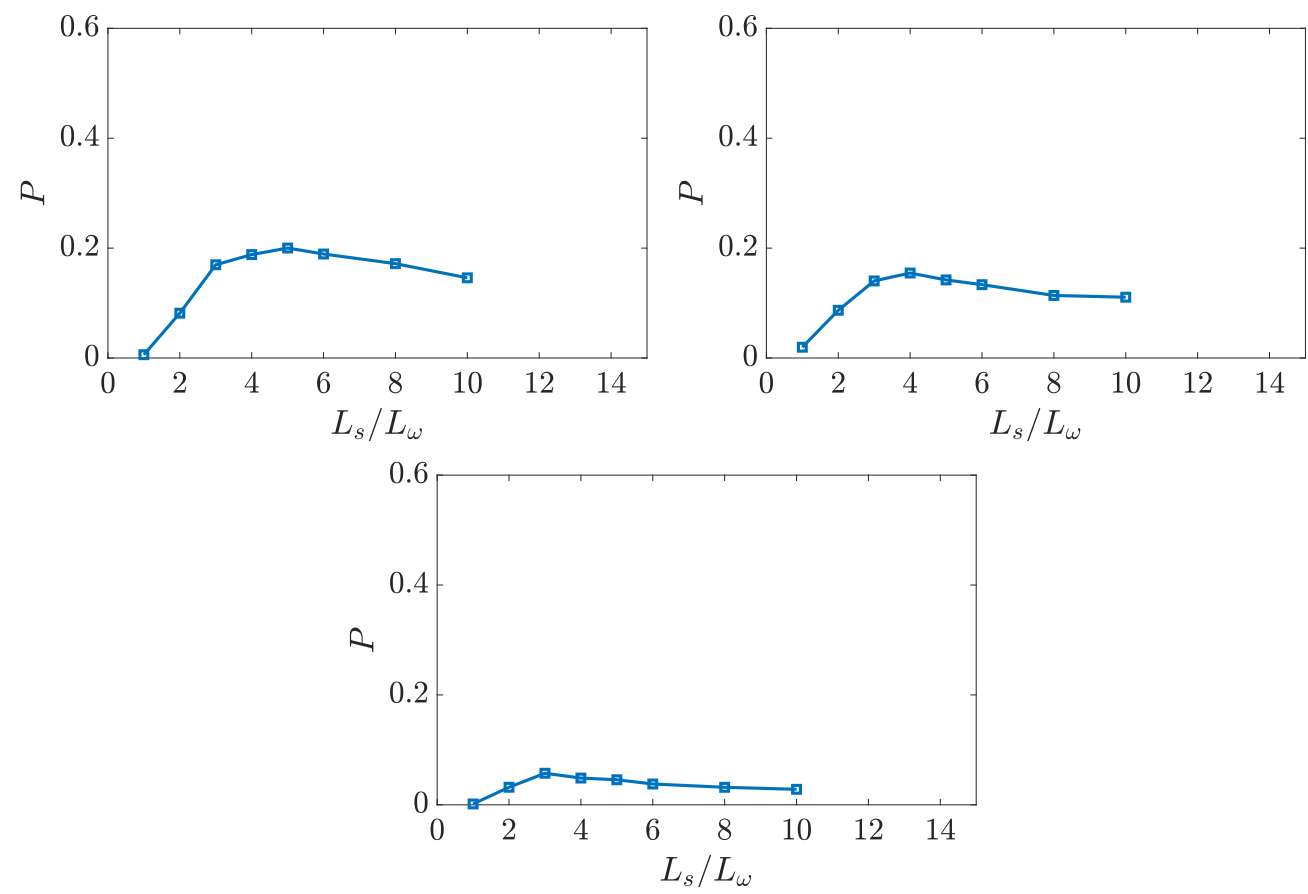

FIG. 12. Probability of perfect alignment between the vorticity at $L_{\omega}$ and extensive principal direction $\alpha$ of the strain rate. Three time instances are shown for the head-on quenching flame with detailed chemistry; $\delta_{z} / s_{L}$ (top left), $2.4 \delta_{z} / s_{L}$ (top right) and $8.2 \delta_{z} / s_{L}$ (bottom).

at $t=6 \delta_{z} / s_{L}$. In the situation, when the flame is at an advanced stage of flame quenching, $P$ tends to vanish. In the detailed chemistry case $\mathrm{G}$, the probability of alignment behaves in a similar manner to that of simple chemistry cases, as shown in figure. 12 .

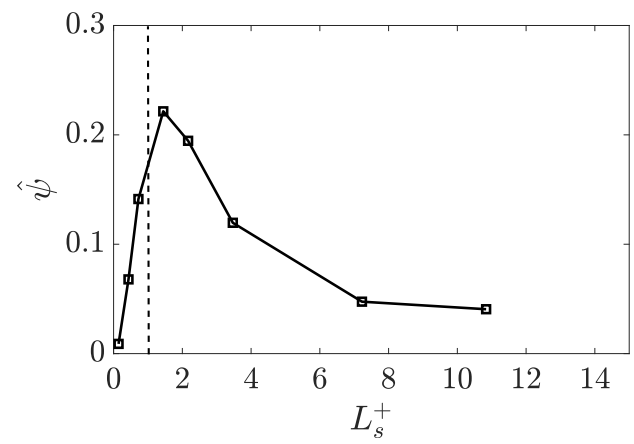

FIG. 13. Surface averaged tangential strain rate from eddies of scale $L_{s}^{+}$normalised by total contribution in the planar flame (case-H). The vertical dashed line indicates $L_{s}^{+}=1$. 

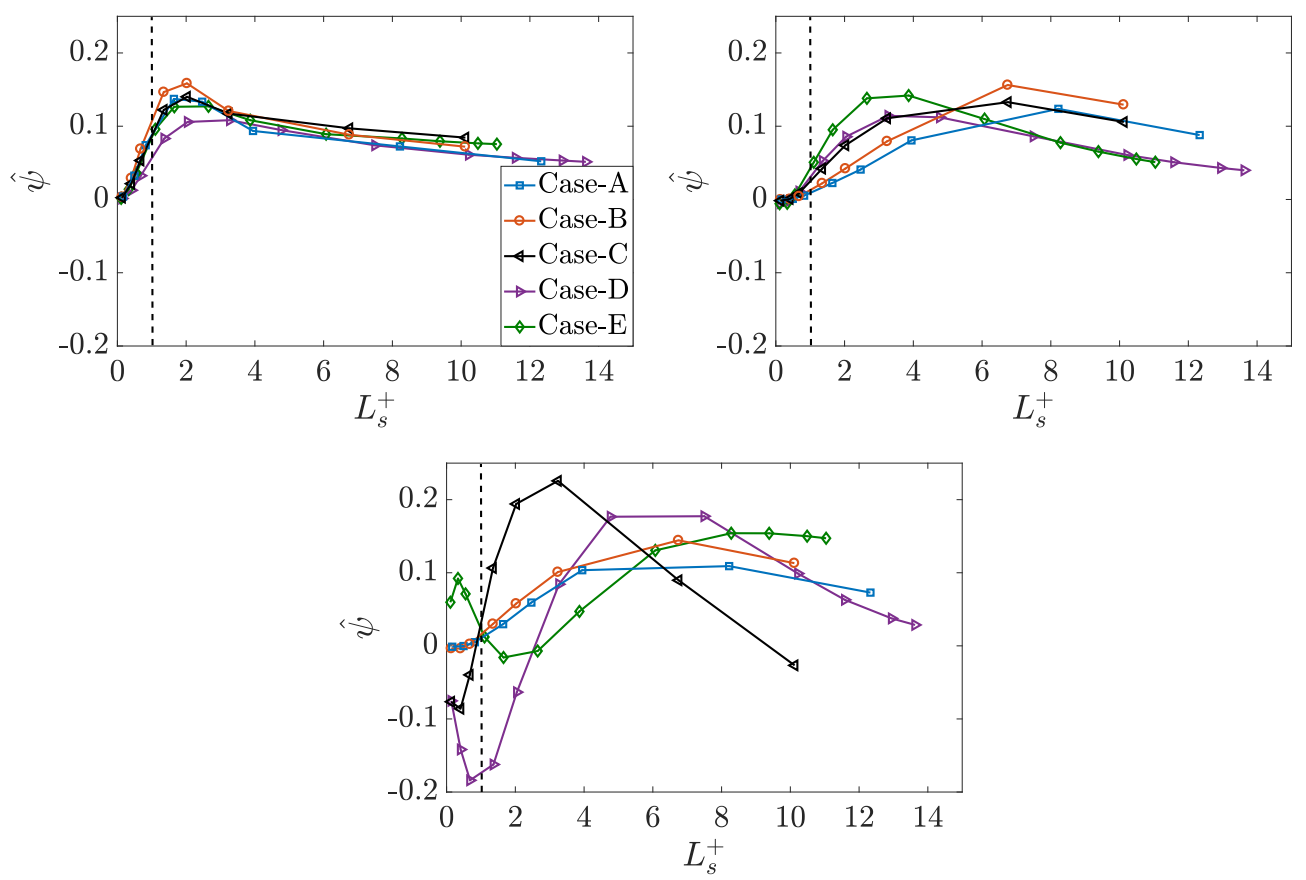

FIG. 14. Surface averaged tangential strain rate from eddies of scale $L_{s}^{+}$normalised by total contribution. Three time instances are shown for simple chemistry head-on quenching flame; $\delta_{z} / s_{L}$ (top left), $4 \delta_{z} / s_{L}$ (top right) and $6 \delta_{z} / s_{L}$ (bottom). The vertical dashed line indicates $L_{s}^{+}=1$.

\section{B. Multiscale analysis of tangential strain rate}

The bandpass filtering technique is used to determine the tangential strain contribution for eddies of scale $L_{s}$ via $a_{T}^{L_{s}}=\left(\delta_{i j}-n_{i} n_{j}\right) S_{i j}^{L_{s}}$ and its surface averaged value is determined as :

$$
\psi\left(L_{s}^{+}\right)=\left\langle|\nabla c| a_{T}^{L_{s}^{+}}\right\rangle /\langle\nabla c\rangle
$$

where $L_{s}^{+}=L_{s} / \delta_{t h}$ and $\psi_{\text {int }}=\int_{0}^{\infty} \psi d L_{s}^{+}$gives the surface-averaged contributions from all the eddies contained in the flow. Figure 13 shows the variation of $\hat{\psi}=\psi / \psi_{\text {int }}$, with $L_{s}^{+}$ for case-H. The peak value for $\hat{\psi}$ is obtained for $2 \leq L_{s}^{+} \leq 3$ which is consistent with the earlier findings for freely-propagating statistically planar flames at high Karlovitz number ${ }^{14}$. A similar qualitative behaviour is observed for the head-on quenching flames (as shown in Figure 14) at $t=1 \delta_{z} / s_{L}$ and $t=4 \delta_{z} / s_{L}$ but at $t=6 \delta_{z} / s_{L}$ when the flame-wall interaction is in an advanced stage, negative values of $\hat{\psi}$ are observed at small values of $L_{s}^{+}$for cases C-E. The negative values of tangential strain rate have been observed at advanced stages of flame quenching in the earlier studies on head-on quenching ${ }^{41}$ (see figure 4 of $^{41}$ ) and occurs 

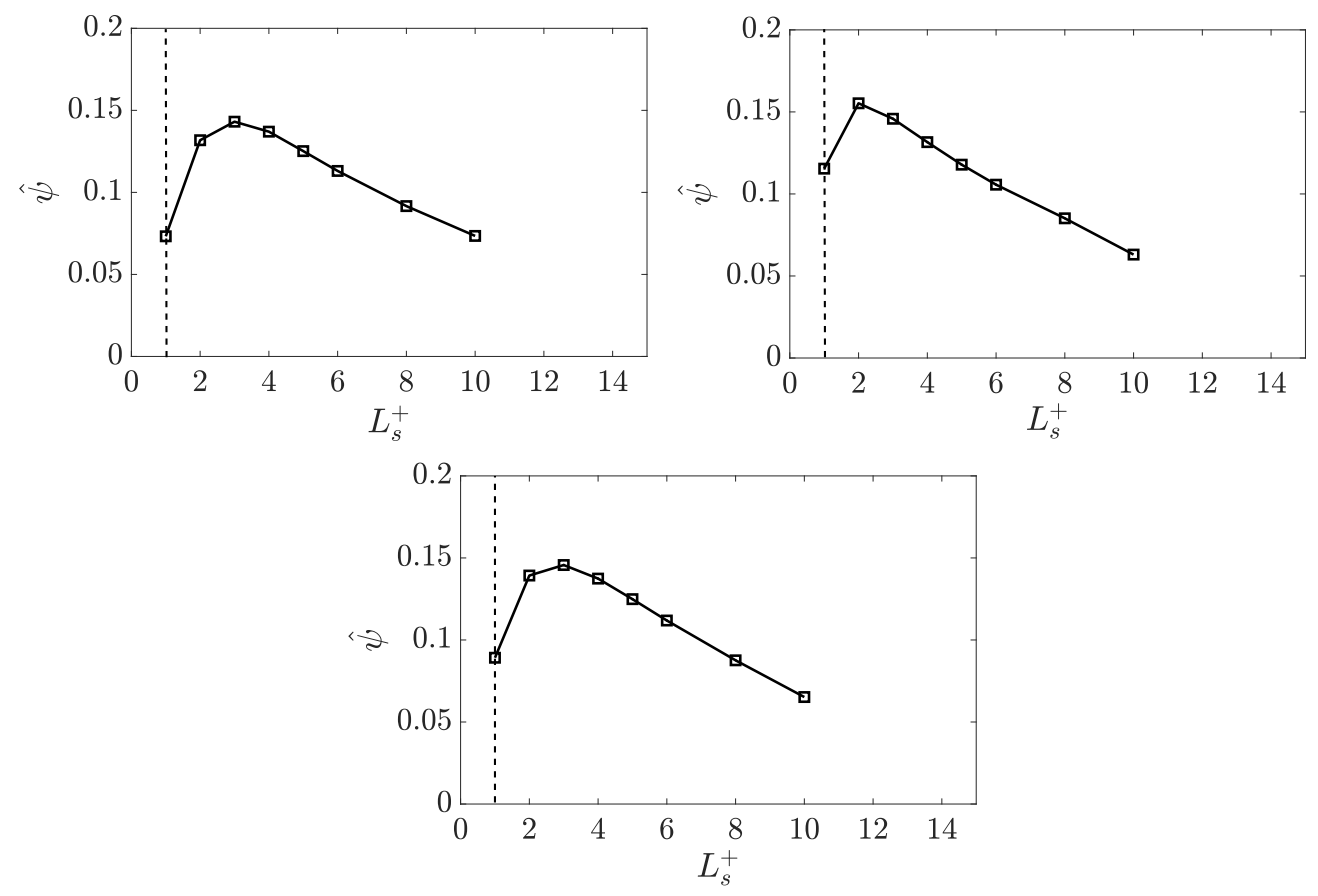

FIG. 15. Surface averaged tangential strain rate from eddies of scale $L_{s}^{+}$normalised by total contribution. Three time instances are shown for the detailed chemistry head-on quenching flame; $\delta_{z} / s_{L}$ (top left), $2.4 \delta_{z} / s_{L}$ (top right) and $8.2 \delta_{z} / s_{L}$ (bottom). The vertical dashed line indicates $L_{s}^{+}=1$.

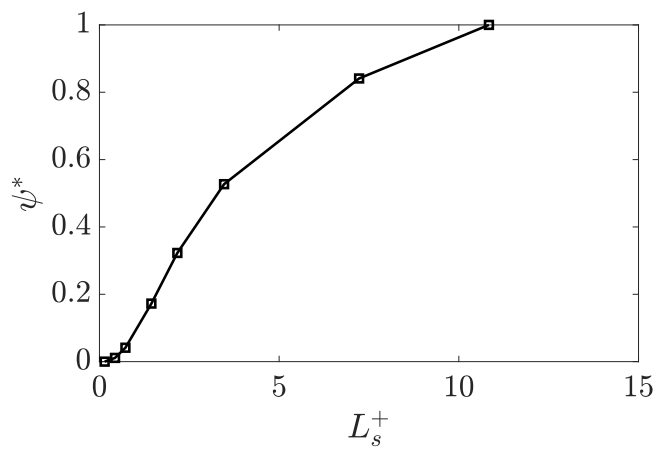

FIG. 16. Cumulative integral of the surface averaged tangential strain rate from eddies of scale $L_{s}^{+}$ for the planar flame (case-H).

due to the negative dilatation rate at later stages of the flame quenching as demonstrated by Lai et al..$^{34}$ (see figure $7 \mathrm{in}^{34}$ ). The detailed chemistry case-G shows qualitatively similar trends for $\hat{\psi}$ as shown in figure 15. The value of $\hat{\psi}$ decreases for large values of $L_{s}^{+}$(e.g. $L_{s}^{+} \approx 7$ ) for all head on quenching flames, which implies that eddies with $4 \leq L_{s}^{+} \leq 7$ have a 
significant influence on flame straining in head-on quenching flames. This can be confirmed further by the cumulative integral $\psi^{*}=\int_{0}^{L_{s}^{+}} \hat{\psi} d L_{s}^{+}$. Figure 16 shows $\psi^{*}$ for the planar flame case- $\mathrm{H}$ and it can be noticed that the scales smaller than $L_{s}^{+}=1.5$ contribute less than $10 \%$ to $\psi^{*}$, whereas eddies larger than $L_{s}^{+}=8$ contribute less than $20 \%$, which is consistent with the earlier findings for freely-propagating planar flames ${ }^{14}$. Similar behaviour can be seen for head-on quenching flames at $t=\delta_{z} / s_{L}$. At later times (e.g. $t=4 \delta_{z} / s_{L}$ and $6 \delta_{z} / s_{L}$ ) the contribution to $\psi^{*}$ from scales smaller than $L_{s}^{+}=3$ increases to almost $20 \%$ for higher turbulence intensity flames which implies that the small scales of turbulence have a much higher influence on the flame in the near-wall region. Similar qualitative trends have been observed in the detailed chemistry case-G as shown in figure 18.
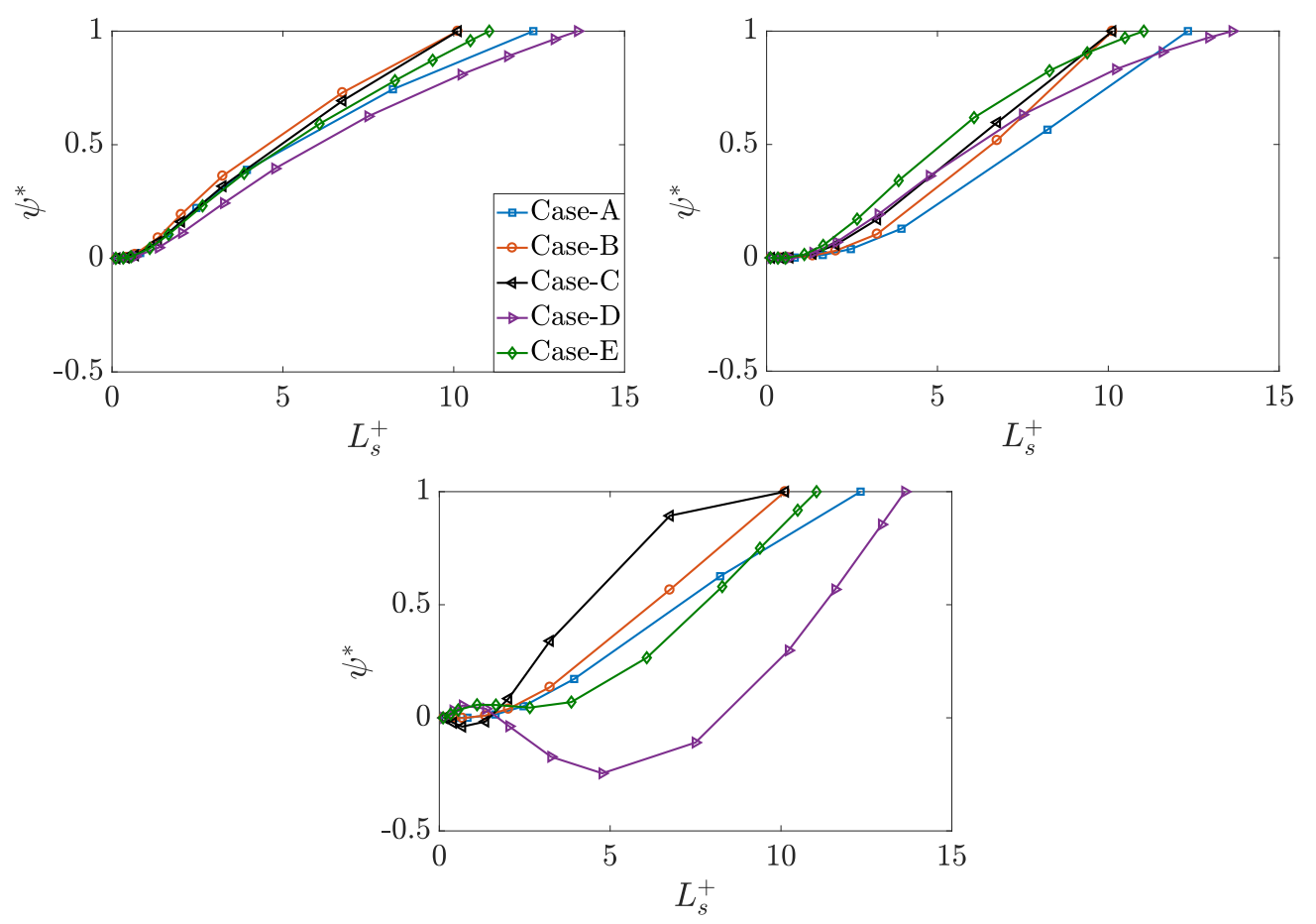

FIG. 17. Cumulative integral of the surface averaged tangential strain rate from eddies of scale $L_{s}^{+}$. Three time instances are shown for the head-on quenching flames with simple chemistry (cases A-E); $\delta_{z} / s_{L}$ (top left), $4 \delta_{z} / s_{L}$ (top right) and $6 \delta_{z} / s_{L}$ (bottom).

The upper and lower cut-off scales can be defined as $l_{10}^{+}$and $l_{p}^{+}$respectively, where $l_{10}^{+}$ corresponds to the length scale at $\psi^{*}=0.1$ and $l_{p}^{+}$corresponds to the length scale at the peak value of $\hat{\psi}$. The eddies smaller than $l_{10}^{+}$contribute less than $10 \%$ or smaller to the total tangential strain rate experienced by the flame. The value of $l_{10}^{+}$changes in time for 

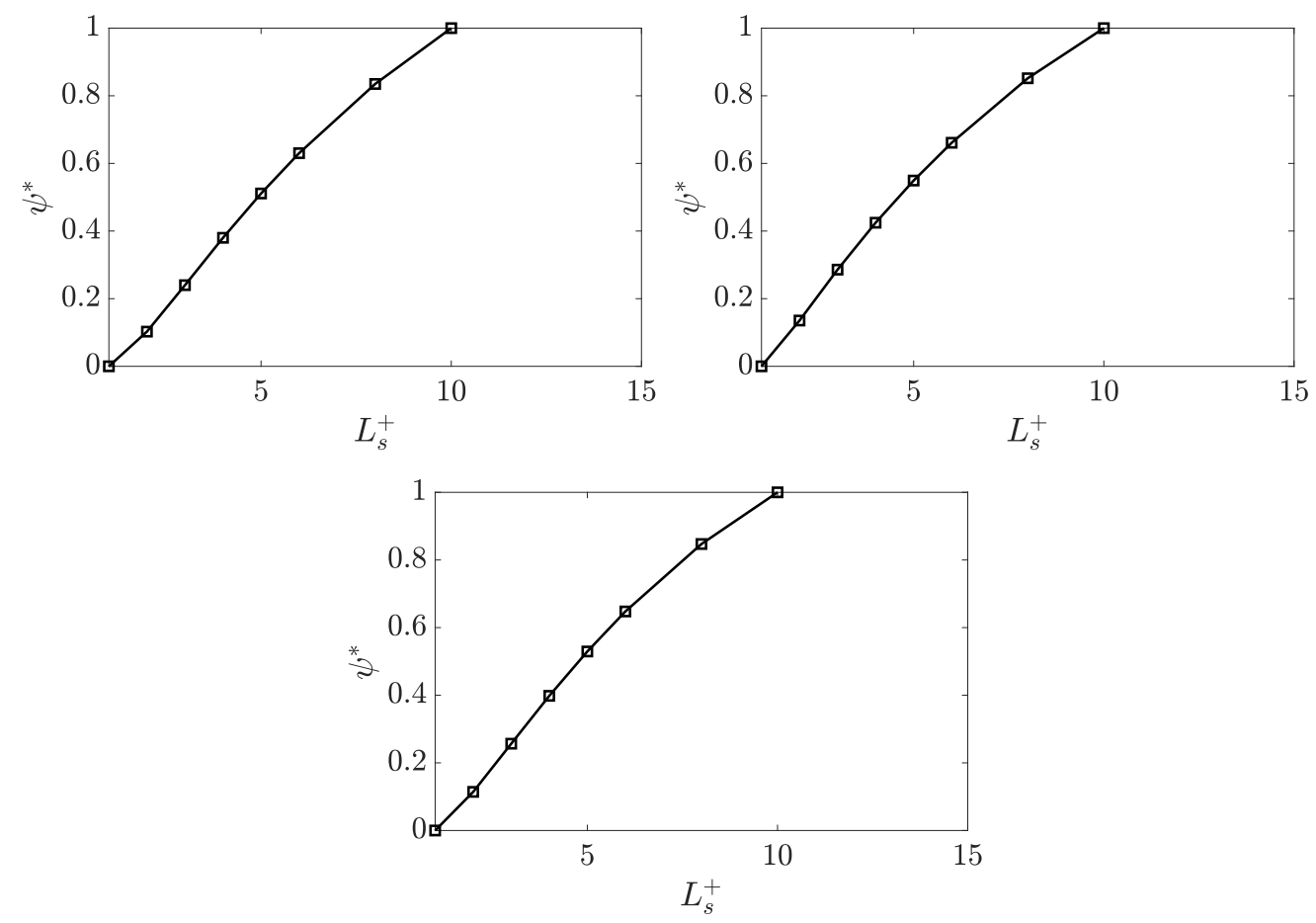

FIG. 18. Cumulative integral of the surface averaged tangential strain rate from eddies of scale $L_{s}^{+}$. Three time instances are shown for the head-on quenching flame with detailed chemistry (cases-G); $\delta_{z} / s_{L}$ (top left), $2.4 \delta_{z} / s_{L}$ (top right) and $8.2 \delta_{z} / s_{L}$ (bottom).

all of the cases investigated in this work. In cases $\mathrm{A}, \mathrm{B}, \mathrm{C}$ and $\mathrm{G}, l_{10}^{+}$initially increases with time and then decays, whereas in cases $\mathrm{D}$ and $\mathrm{E} l_{10}^{+}$shows a monotonic increase with time. The difference in behaviour of cases D and E exists due to the earlier initiation of flame-wall interaction (as shown in figures 4 and 5) which leads to changes in the vortical structure as discussed in the previous sections. The values for $l_{p}^{+}$are of the same order of magnitude to that of $l_{10}^{+}$and also show similar trends to that of $l_{10}^{+}$as well. In the case of head-on quenching $l_{10}$ remains an order of magnitude greater than the Gibson length scale $^{36}\left(l_{G}=s_{L}^{3} / \widetilde{\epsilon}\right.$, where $\epsilon$ is the turbulence dissipation rate), but scales with the length scale proposed by Roberts et al. ${ }^{39}\left(l_{R} / \delta_{t h}=2\left(\widetilde{k}^{0.5} / s_{L}\right)^{-3 / 4}\left(\left(\widetilde{k}^{3 / 2} / \widetilde{\epsilon}\right) / \delta_{t h}\right)^{1 / 4}\right)$ as exemplarily shown for head-on quenching cases in figure 19 for a location corresponding to $\widetilde{T}=0.9$. This implies that in a LES calculation resolving $l_{p}^{+}$or $l_{10}^{+}$would be sufficient to resolve the tangential strain rate term in the context of FSD transport equation. Furthermore, the inner cut-off scale $l_{p}$ has been found to be of the order of flame thickness $\delta_{t h}$ which is consistent with previous analysis in the context of $\mathrm{FSD}^{12,20,27}$ and scalar dissipation rate ${ }^{15}$ closures. 


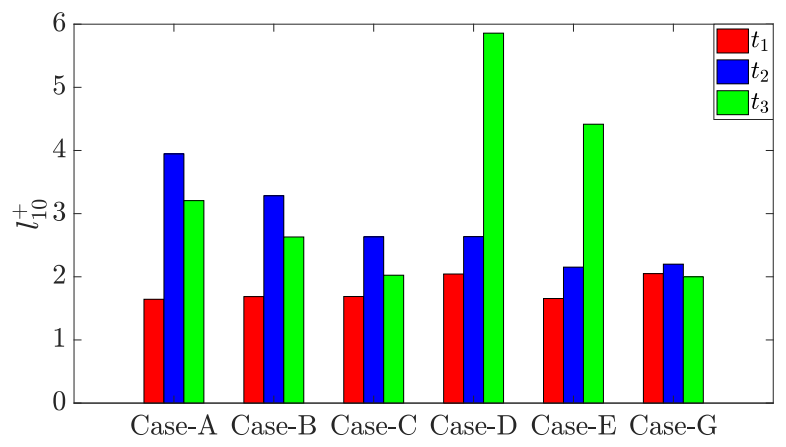

(a) $l_{10}^{+}$

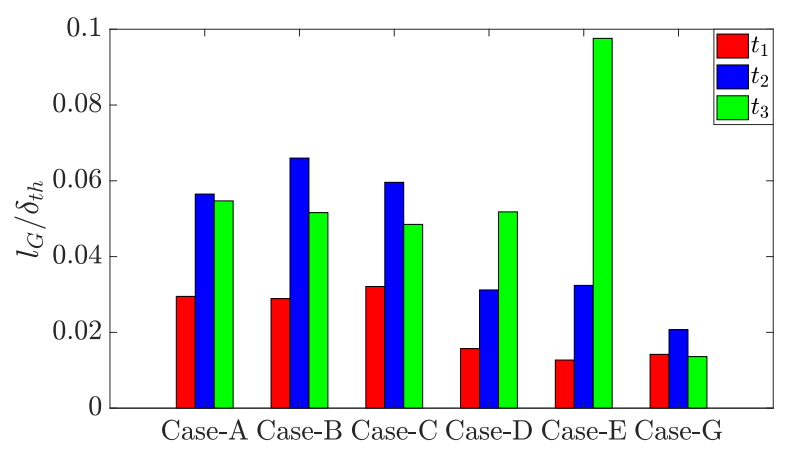

(c) $l_{G}$

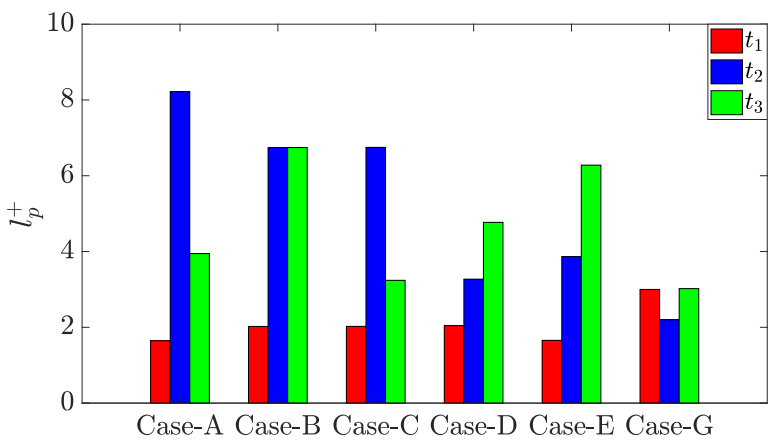

(b) $l_{p}^{+}$

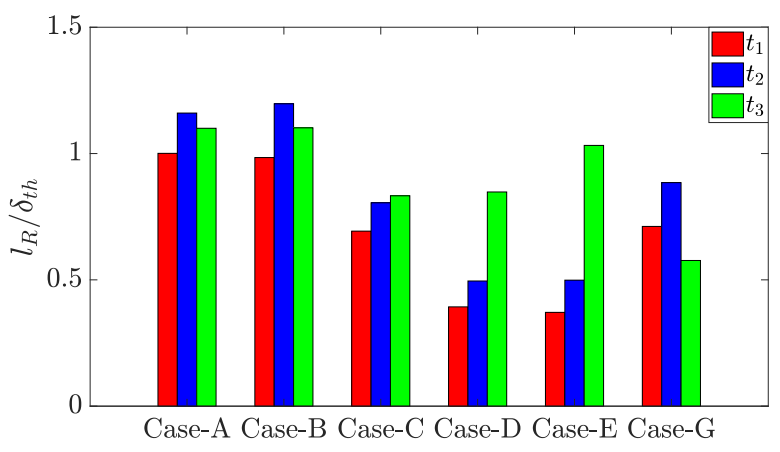

(d) $l_{R}$

FIG. 19. Comparison of various cutoff scales at different times for head-on quenching cases. $t_{1}, t_{2}$ and $t_{3}$ imply $\delta_{z} / s_{L}, 4 \delta_{z} / s_{L}$ and $6 \delta_{z} / s_{L}$ for cases A-E and $\delta_{z} / s_{L}, 2.4 \delta_{z} / s_{L}$ and $8.2 \delta_{z} / s_{L}$ for cases $\mathrm{G}$ respectively.

\section{CONCLUSIONS}

Multiscale analysis of head-on quenching turbulent premixed flames has been performed using bandpass filtering of DNS data based on simple 1-step and skeletal (16 species 25 reaction mechanism ${ }^{42}$ ) chemical mechanisms at different turbulence intensities. A freely propagating statistically planar flame subjected to isotropic forced turbulence has also been investigated to demonstrate the differences between head-on quenching and freely propagating planar flames. The bandpass filtering technique is used to determine the influence of eddies of a given scale $L_{s}$ in the turbulence spectrum. The statistics of the alignment between vorticity and the principal directions of strain rate are investigated at different length scales. It has been observed that when the flames are away from the wall the vortical structures of scale $L_{\omega}$ are stretched by large eddies of scale around $2 L_{\omega}$ to $4 L_{\omega}$ which is consistent with the earlier findings for freely-propagating statistically planar flames ${ }^{14}$ and 
non-reacting flows ${ }^{35}$. It has also been observed that the vorticity field in the near-wall region is altered by the presence of the flame during flame quenching and under this condition the influence of non-local/ background strain rate field vanishes.

The statistics of surface-averaged tangential strain rate is calculated to determine the influence of different scales of turbulence. It has been found that the eddies smaller than $3 \delta_{t h}$ contribute less than $10 \%$ of the total surface-averaged tangential strain rate, while eddies larger than $8 \delta_{t h}$ contribute less than $20 \%$ of the total surface-averaged tangential strain rate when the flame is away from the wall. These findings are consistent with those of freely-propagating premixed turbulent statistically planar flames ${ }^{14}$. In the case when flames start to interact with the wall, the contribution from the eddies smaller than $3 \delta_{t h}$ contribute more than $20 \%$ to the surface-averaged tangential strain rate for high values of turbulence intensity. It is also found that the choice of chemical mechanism does not influence the underlying fluid mechanical processes involved in vortex stretching. This is in agreement with the earlier experimental findings ${ }^{21,22}$.

The cases considered in this work deal with atmospheric flames, which is consistent with all the previous DNS investigations for flame-wall interaction ${ }^{1,2,7,16,37}$. The variation in pressure affects the laminar burning velocity and the flame thickness. An increase in pressure gives rise to a reduction in flame thickness, which makes the grid resolution requirements prohibitively expensive for high-pressure flames as obtained in SI engines and gas turbines. This difficulty is exacerbated further in the presence of walls for high-pressure flames. A recent analysis by Klein et al. ${ }^{25}$ revealed that the statistics of strain rate and scalar gradient normalised by the appropriate combinations of laminar burning velocity and thermal flame thickness remain both qualitatively and quantitatively similar for premixed flames at different pressure levels under a given set of values of $s_{L}$ and $\delta_{z}$. As the current work focuses on multiscale analysis, which principally deals with strain rate and vorticity statistics, the qualitative nature of the findings is unlikely to be affected by the pressure variation. However, the influence of pressure on multi-scale analysis of flame-wall interaction needs to be explored further in the future investigations. The present findings have important implications for the subgrid scale strain rate term modelling in the context of the flame surface density (FSD) transport equation for large eddy simulation (LES). The results in this work suggest that the subgrid strain rate modelling (and the inaccuracies associated with it) might not have a major implication for LES filter sizes of the order of few multiples 
of $\delta_{t h}$ for flames away from the wall. However, subgrid modelling in the near-wall region would require both higher resolution and a more robust closure for the strain rate term to ensure high fidelity of the simulations, as the cut-off scales decrease close to the wall.

\section{ACKNOWLEDGEMENTS}

The authors are grateful to EPSRC (EP/P022286/1) for financial support. The computational support was provided by ARCHER (EP/K025163/1) and the HPC facility at Newcastle University (Rocket). NAKD acknowledges the support of the Qualcomm European Research Studentship.

\section{REFERENCES}

${ }^{1}$ T. M. Alshaalan and C. J. Rutland. Turbulence, scalar transport, and reaction rates in flame-wall interaction. Proc. Combust. Inst., 27(1):793-799, jan 1998.

${ }^{2}$ T. M. Alshaalan and C. J. Rutland. Wall heat flux in turbulent premixed reacting flow. Combust. Sci. Technol., 174:135-165, 2002.

${ }^{3}$ W. T. Ashurst, A. R. Kerstein, R. M. Kerr, and C. H. Gibson. Alignment of vorticity and scalar gradient with strain rate in simulated Navier Stokes turbulence. Phys. Fluids, 30(8):2343, 1987.

${ }^{4}$ G. K. Batchelor and A. A. Townsend. Decay of Turbulence in the Final Period. Proc. $R$. Soc. A Math. Phys. Eng. Sci., 194(1039):527-543, nov 1948.

${ }^{5} \mathrm{~B}$. Bobbitt and G. Blanquart. Vorticity transformation in high Karlovitz number premixed flames. Phys. Fluids, 28(1):015101, jan 2016.

${ }^{6}$ B. Bobbitt, S. Lapointe, and G. Blanquart. Vorticity transformation in high Karlovitz number premixed flames. Phys. Fluids, 28(1):015101, 2016.

${ }^{7}$ G. Bruneaux, T. J. Poinsot, and J. H. Ferziger. Premixed flamewall interaction in a turbulent channel flow: budget for the flame surface density evolution equation and modelling. J. Fluid Mech., 349:191-219, oct 1997.

${ }^{8}$ S. M. Candel and T. J. Poinsot. Flame stretch and the balance equation for the flame area. Combust. Sci. Technol., 70(1):1-15, mar 1990. 
${ }^{9}$ R. S. Cant. SENGA2 User Guide, CUED/ A-THERMO/TR67. Technical report, University of Cambridge, Cambridge, United Kingdom, 2012.

${ }^{10}$ R. S. Cant, S. B. Pope, and K. N. C. Bray. Modelling of flamelet surface-to-volume ratio in turbulent premixed combustion. Proc. Combust. Inst., 23(1):809-815, 1991.

${ }^{11}$ N. Chakraborty. Statistics of vorticity alignment with local strain rates in turbulent premixed flames. Eur. J. Mech. - B/Fluids, 46:201-220, jul 2014.

${ }^{12}$ N. Chakraborty and M. Klein. A priori direct numerical simulation assessment of algebraic flame surface density models for turbulent premixed flames in the context of large eddy simulation. Phys. Fluids, 20(8):085108, aug 2008.

${ }^{13}$ O. Colin, F. Ducros, D. Veynante, and T. J. Poinsot. A thickened flame model for large eddy simulations of turbulent premixed combustion. Phys. Fluids, 12(7):1843-1863, jul 2000 .

${ }^{14}$ N. Doan, N. Swaminathan, and N. Chakraborty. Multiscale analysis of turbulence-flame interaction in premixed flames. Proc. Combust. Inst., 36(2):1929-1935, 2017.

${ }^{15}$ Y. Gao, N. Chakraborty, and N. Swaminathan. Algebraic closure of scalar dissipation rate for large eddy simulations of turbulent premixed combustion. Combust. Sci. Technol., 186(10-11):1309-1337, 2014.

${ }^{16}$ A. Gruber, R. Sankaran, E. R. Hawkes, and J. H. Chen. Turbulent flamewall interaction: a direct numerical simulation study. J. Fluid Mech., 658:5-32, sep 2010.

${ }^{17}$ P. E. Hamlington, A. Y. Poludnenko, and E. S. Oran. Interactions between turbulence and flames in premixed reacting flows. Phys. Fluids, 23(12):125111, 2011.

${ }^{18}$ P. E. Hamlington, J. Schumacher, and W. J. A. Dahm. Direct assessment of vorticity alignment with local and nonlocal strain rates in turbulent flows. Phys. Fluids, 20(11):1$5,2008$.

${ }^{19}$ E. R. Hawkes and R. S. Cant. Implications of a flame surface density approach to large eddy simulation of premixed turbulent combustion. Combust. Flame, 126(01):1617-1629, 2001.

${ }^{20}$ K. Hiraoka, Y. Minamoto, M. Shimura, Y. Naka, N. Fukushima, and M. Tanahashi. A Fractal Dynamic SGS Combustion Model for Large Eddy Simulation of Turbulent Premixed Flames. Combust. Sci. Technol., 188(9):1472-1495, 2016.

${ }^{21}$ C. Jainski, M. Rißmann, B. Böhm, and A. Dreizler. Experimental investigation of flame surface density and mean reaction rate during flame-wall interaction. Proc. Combust. Inst., 
36(2):1827-1834, 2017.

${ }^{22}$ C. Jainski, M. Rißmann, S. Jakirlic, B. Böhm, and A. Dreizler. Quenching of Premixed Flames at Cold Walls: Effects on the Local Flow Field. Flow, Turbul. Combust., 100(1):177-196, 2018.

${ }^{23} \mathrm{~K}$. Jenkins and R. Cant. Direct numerical simulation of turbulent flame kernels. In D. Knight and L. Sakell, editors, Recent Advances in DNS and LES: Proceedings of the Second AFOSR Conference, Rutgers - The State University of New Jersey, New Brunswick, USA, pages 191-202. Kluwer, Dordrecht, 1999.

${ }^{24}$ J. Jiménez. Kinematic alignment effects in turbulent flows. Phys. Fluids A, 4(4):652-654, 1992.

${ }^{25}$ M. Klein, D. Alwazzan, and N. Chakraborty. A direct numerical simulation analysis of pressure variation in turbulent premixed Bunsen burner flames-Part 1: Scalar gradient and strain rate statistics. Comput. Fluids, 173:178-188, 2018.

${ }^{26}$ M. Klein, N. Chakraborty, and S. Ketterl. A Comparison of Strategies for Direct Numerical Simulation of Turbulence Chemistry Interaction in Generic Planar Turbulent Premixed Flames. Flow, Turbul. Combust., 99(3-4):955-971, dec 2017.

${ }^{27}$ R. Knikker, D. Veynante, and C. Meneveau. A dynamic flame surface density model for large eddy simulation of turbulent premixed combustion. Phys. Fluids, 16(11):L91, 2004.

${ }^{28}$ J. Lai and N. Chakraborty. A Priori Direct Numerical Simulation Modeling of Scalar Dissipation Rate Transport in Head-On Quenching of Turbulent Premixed Flames. Combust. Sci. Technol., 188(9):1440-1471, sep 2016.

${ }^{29}$ J. Lai and N. Chakraborty. Effects of Lewis Number on Head on Quenching of Turbulent Premixed Flames: A Direct Numerical Simulation Analysis. Flow, Turbul. Combust., 96(2):279-308, mar 2016.

${ }^{30}$ J. Lai, N. Chakraborty, and A. N. Lipatnikov. Statistical behaviour of vorticity and enstrophy transport in head-on quenching of turbulent premixed flames. Eur. J. Mech. B/Fluids, 65:384-397, 2017.

${ }^{31}$ J. Lai, M. Klein, and N. Chakraborty. Assessment of Algebraic Flame Surface Density Closures in the Context of Large Eddy Simulations of Head-On Quenching of Turbulent Premixed Flames. Combust. Sci. Technol., 189(11):1-26, 2017.

${ }^{32}$ J. Lai, M. Klein, and N. Chakraborty. Direct Numerical Simulation of Head-On Quenching of Statistically Planar Turbulent Premixed Methane-Air Flames Using a Detailed Chemical 
Mechanism. Flow, Turbul. Combust., pages 1-19, 2018.

${ }^{33}$ J. Lai, A. Moody, and N. Chakraborty. Turbulent kinetic energy transport in head-on quenching of turbulent premixed flames in the context of Reynolds Averaged Navier Stokes simulations. Fuel, 199:456-477, 2017.

${ }^{34}$ J. Lai, D. H. Wacks, and N. Chakraborty. Flow topology distribution in head-on quenching of turbulent premixed flame: A Direct Numerical Simulation analysis. Fuel, 224(February 2017):186-209, 2018.

${ }^{35}$ T. Leung, N. Swaminathan, and P. Davidson. Geometry and interaction of structures in homogeneous isotropic turbulence. J. Fluid Mech., 710:453-481, 2012.

${ }^{36}$ N. Peters. Turbulent Combustion. Cambridge University Press, 2000.

${ }^{37}$ T. J. Poinsot, D. Haworth, and G. Bruneaux. Direct simulation and modeling of flame-wall interaction for premixed turbulent combustion. Combust. Flame, 95(1):118-132, 1993.

${ }^{38}$ T. J. Poinsot, D. Veynante, and S. M. Candel. Quenching processes and premixed turbulent combustion diagrams. J. Fluid Mech., 228:561-606, jul 1991.

${ }^{39}$ W. L. Roberts, J. F. Driscoll, M. C. Drake, and L. P. Goss. Images of the quenching of a flame by a vortex-To quantify regimes of turbulent combustion. Combust. Flame, 94(1-2), 1993.

${ }^{40}$ R. S. Rogallo. Numerical Experiments in Homogeneous Turbulence. Technical report, NASA AMES, 1981.

${ }^{41}$ J. Sellmann, J. Lai, A. M. Kempf, and N. Chakraborty. Flame surface density based modelling of head-on quenching of turbulent premixed flames. Proc. Combust. Inst., 36(2):1817-1825, 2017.

${ }^{42}$ M. D. Smooke and V. Giovangigli. Premixed and nonpremixed test problem results, pages 29-47. Springer Berlin Heidelberg, Berlin, Heidelberg, 1991.

${ }^{43}$ H. Wang, E. R. Hawkes, and J. H. Chen. Turbulence-flame interactions in DNS of a laboratory high Karlovitz premixed turbulent jet flame. Phys. Fluids, 28(9):095107, 2016.

${ }^{44}$ C. S. Yoo and H. G. Im. Characteristic boundary conditions for simulations of compressible reacting flows with multi-dimensional, viscous and reaction effects. Combust. Theory Model., 11(2):259-286, apr 2007. 